\title{
Gas properties in the disc of NGC 891 from Herschel far-infrared spectroscopy
}

\author{
Thomas M. Hughes \\ Astronomical Observatory, Ghent University, Belgium \\ email: thomas .hughes@ugent.be
}

\begin{abstract}
We investigate the physical properties of the interstellar gas in the nearby edge-on spiral galaxy NGC 891, using Herschel PACS/SPIRE observations of the most important farinfrared (FIR) cooling lines - [CII] $158 \mu \mathrm{m}$, [NII] 122, $205 \mu \mathrm{m}$, [OI] 63, $145 \mu \mathrm{m}$, and [OIII] $88 \mu \mathrm{m}$ - obtained as part of the Very Nearby Galaxy Survey (P. I.: C. D. Wilson). We compare our observations to the predictions of a photo dissociation region (PDR) model to determine the gas density, $n$, and the strength of the incident FUV radiation field, $G_{0}$, on a pixel-by-pixel basis. The majority of PDRs in NGC 891's disc exhibit properties similar to the physical conditions found in the spiral arm and inter-arm regions of the face-on M51 galaxy. We estimate a stronger FUV field in the far north-eastern side than compared to the rest of the disc.
\end{abstract}

Keywords. galaxies: individual: NGC 891 - galaxies: spiral - ISM: lines and bands

\section{Importance of optical depth effects}

We determine the gas properties from the FIR cooling line and TIR emission, estimated from 24, 100 and $160 \mu \mathrm{m}$ maps (see Hughes et al. 2014), using the Kaufman et al. (2006) PDR model. Radial trends in $n$ and $G_{0}$ found in face-on galaxies, e.g. M51 (Parkin et al. 2013), are only reproduced by correcting the [OI] $63 \mu \mathrm{m}$ line flux to account for increasing optical thickness towards the center (Fig. 1), implying such effects must be considered when interpreting observations of highly inclined systems (Hughes et al. submitted).

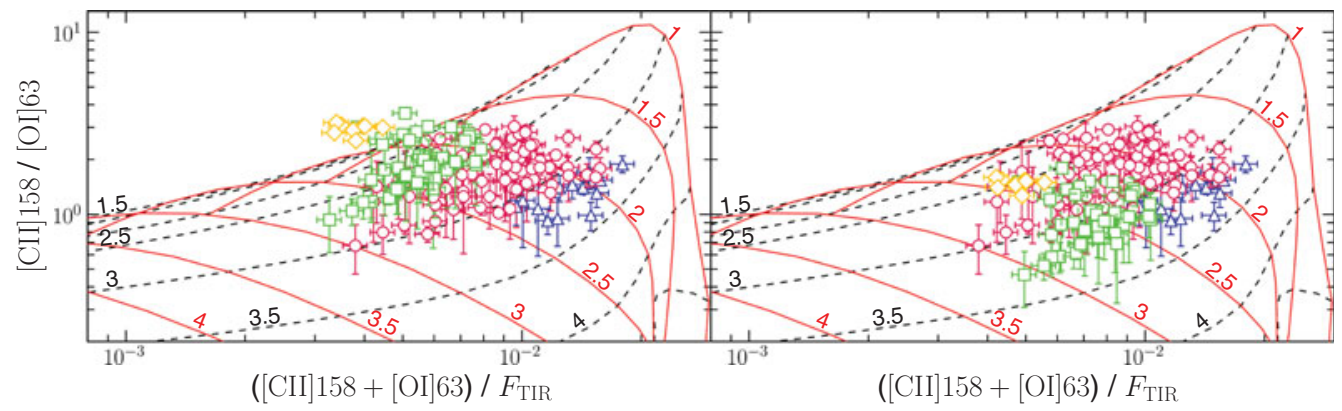

Figure 1. The $[\mathrm{CII}] /[\mathrm{OI}] 63$ versus $([\mathrm{CHI}]+[\mathrm{OI}] 63) / F_{\mathrm{TIR}}$ ratio diagnostic diagram, in which we superimpose our adjusted observations onto grid-lines of constant $\log n$ (dashed lines) and $\log$ $G_{0}$ (solid lines) determined from the PDR model. The points correspond to pixels covering the nucleus (diamonds), mid-plane (squares) and at increasing radial distances (circles/triangles). We contrast a constant (left) and varying correction (right) to the [OI] $63 \mu \mathrm{m}$ emission.

\section{References}

Hughes, T. M., Baes, M., Fritz, J., et al. 2014, A\& $A$, 565, 4

Hughes, T. M., Foyle, K., Schirm, M. R. P., et al. 2014, A\&A, submitted

Kaufman, M. J., Wolfire, M. G., \& Hollenbach, D. J. 2006, ApJ, 644, 283

Parkin, T. J., Wilson, C. D., Schirm, M. R. P., et al. 2013, A\&̊A, 550, 114 